\title{
ARTIGOORIGINAL
}

\section{Automatização do protocolo de aquisição e pré-processamento de imagens térmicas do sensor TIRS do satélite Landsat-8}

\section{Automatization of protocols for the acquisition and preprocessing of thermal images using sensor TIRS of Landsat-8}

Luiz Rodrigo Gonzaga de Oliveira ${ }^{(1}$ and Sérgio Barros de Sousa ${ }^{2}$

${ }^{1}$ Universidade Estadual do Piauí, ${ }^{2}$ Universidade Estadual do Piauí

*luizrodrigo46@hotmail.com; sergiobarros@pq.cnpq.br

Recebido: 27/07/2019. Revisado: 20/04/2020. Aceito: 14/10/2020.

\section{Resumo}

A coleta de imagens térmicas de satélites para o estudo de uma determinada região é feita acessando sistemas na internet como o Serviço de Levantamento Geológico Americano (USGS). A cada novo estudo etapas de aquisição e recorte de imagens de satélites para uma determinada região são feitas de forma individualizada, sendo um protocolo bem comum em pesquisas de imagens de satélites orbitais. Este trabalho apresenta um protocolo automatizado de aquisição e pré-processamento de imagens de satélites do sensor térmico TIRS do Landsat-8 que possa contribuir para a otimização da performance de pesquisas geográficas. Para o desenvolvimento desse protocolo foram analisados os processos manuais comuns de coleta e recorte em pesquisas geográficas que fazem o uso de imagens térmicas do Landsat-8. Foi observado que é possível obter e pre-processar imagens térmicas do Landsat- 8 automaticamente, possibilitando a junção com outras técnicas automatizadas e acoplamento com plataformas SIG que contenham processamento térmico de regiões.

Palavras-Chave: Sensoriamento Remoto; Sistemas Geográficos; Landsat-8; Imagens de satélites; web crawler.

\begin{abstract}
The collection of thermal images of satellites to study a region is made accessing systems on the Internet such as the US Geological Survey (USGS). Every study of satellite images the acquisition and clipping stages for a region is made in an individualized way, being a common protocol in researches of orbital satellite images. In order to contribute to the optimization of geographic researches performance, this work presents an automated protocol for satellite image acquisition and preprocessing of the Landsat-8 TIRS thermal sensor. To develop this protocol, were analyzed common manual processes of acquisition and clipping in geographic researches that uses Landsat- 8 thermal images. It has been found possible to pre-process the Landsat- 8 digital thermocouples automatically, enabling a combination with others automated technologies and coupling with others geographic information systems that contain thermal region processing.
\end{abstract}

Keywords: Remote sensing; Geographic Systems; Landsat-8; Satellite images; Web Crawler. 


\section{Introdução}

A busca de dados para estudos e análises geográficas provenientes de satélites orbitais tem sido nos últimos anos executada com frequência, por conta da disponibilização gratuita dos dados através de plataformas de acesso público. A partir de dados geográficos gerados por essas plataformas, surgiram com o tempo uma grande quantidade de tecnologias que compõe um conjunto de soluções que proporcionam uma maior facilidade em estudos e tomada de decisões nesta área.

As tecnologias geográficas com foco no Sensoriamento Remoto e nos Sistemas de Informações Geográficas, estão sendo amplamente integradas. Atualmente o sensoriamento remoto vem ganhando bastante destaque nos estudos que incluem a análise geográfica, por conta do aprimoramento de hardwares e softwares, utilizando variados sensores em estruturas terrestres, aéreas e orbitais, sem o contato direto com os elementos terrestres. O Thermal Infrared Sensor (TIRS) do satélite Landsat- 8 é um dos recursos remotos que produz dados na faixa do infravermelho termal, que depois de processadas geram imagens em escalas de cinza. Em sequência, a partir dessas imagens é possível fazer um processamento de zonas de calor por meio de algum Sistema de Informação Geográfico, como o QGIS, que é um software com várias ferramentas para o manejo e análise de dados geográficos.

Essas técnicas de acesso manual a imagens dos sensores térmicos de satélites vem sendo utilizadas amplamente por trabalhos de pesquisa que coletam dados e fazem uso de técnicas de sensoriamento remoto orbital e suborbital; merecendo destaque para Rao (1972), que foi o primeiro a demonstrar que as áreas urbanas poderiam ser identificadas por meio de análises de dados na faixa do infravermelho termal adquiridos por um satélite. Pesquisadores como Fialho (2009), Sousa and Júnior (2012), Nascimento and Oliveira (2011), entre outros, também usaram sistemas de sensoriamento remotos infravermelhos termais para documentar o efeito de ilha de calor urbana, assim como extrair informação de temperatura da superfície de distintos materiais.

As imagens de satélites podem ser coletados gratuitamente, graças ao projeto Landsat que teve início na segunda metade da década de 60 , com o intuito de fazer observações dos recursos naturais terrestres. Até então foram lançados 8 satélites, com vários tipos de sensores e com a resolução temporal de 16 dias. Todas as missões do projeto Landsat foram administrados pela National Aeronautics and Space Administration (NASA) e U.S. Geological Survey (USGS).

A USGS fornece uma plataforma chamada Earth Explorer, para a consulta e aquisição de dados de uma região de acordo com pontos no mapa. A coleta de uma imagem de satélite para estudo de uma região é comum em pesquisas geográficas; após essa coleta é feito um recorte da imagem com base em um arquivo específico com vários pontos que delimitam a região de interesse. 0 recorte é necessário nos estudos geográficos pois 0 Landsat retira faixas de fotos extensas das regiỗes do plano terrestre sendo necessário restringir o processamento da imagem a áreas específicas para reduzir o tempo para analisar a imagem. Depois de recortar a imagem é feito um processamento e análise deste recorte, essas etapas formam um protocolo comum em qualquer pesquisa geográfica que utiliza imagens de satélites.

Os protocolos de coleta e recorte de uma imagem de satélite são feitos manualmente a cada novo estudo, para uma determinada área delimitada, podendo demandar um certo tempo para o pesquisador, necessitando uma complexa curva de aprendizado das ferramentas e dos métodos de extração da área, ou, a repetição periódica das etapas, tornando o processo de aquisição de dados extremamente cansativo.

Mozgovoy et al. (2019), indica que organizações governamentais europeias já utilizam a detecção de imagens como meio de predição de desmatamentos evidenciando que a automatização desse processo pode ampliar sobremaneiramente o tempo de identificação de possíveis eventos que exigem respostas rápidas do poder público.

Diante disso, o presente trabalho tem por objetivo padronizar um protocolo de acesso automatizado em tempo real à base de imagens do sensor termal do satélite LandSat-8 e com isso contribuir para a otimização da performance em pesquisas geográficas ao permitir um meio padronizado para coletar os dados periodicamente ao tempo em que forem sendo disponibilizadas, processando e exibindo as imagens já recortadas através de uma interface web, mitigando assim as dificuldades para $o$ acesso e tratamento destas imagens.

\section{Referencial Teórico}

\subsection{Geotecnologias}

As geotecnologias podem compor um conjunto de tecnologias associadas à aquisição, armazenamento, préprocessamento e produção de informações de maneira georreferenciada. A geotecnologia agrega as áreas de Sensoriamento Remoto, Sistemas de Informações Geográficas (SIG), Cartografia Digital e Sistema de Posicionamento Global (GPS) (Matias, 2001).

$\mathrm{O}$ avanço dos instrumentos computacionais em conjunto aos métodos automatizados de análise das informações das geotecnologias tem proporcionado muitas contribuições para a ampliação das categorias de estudo do espaço geográfico, gerando novos conhecimentos do ambiente e das variáveis atuantes na dinâmica de transformação gerada pelo homem (Souza, 2015)

Desta maneira, as geotecnologias podem ser utilizadas para o estudo da paisagem, incluindo a topografia, a hidrografia, a geologia, a geomorfologia e outras variáveis ambientais, assim como analisar e auxiliar na prevenção e no monitoramento de desastres ambientais e aqueles causados pela atividade humana (Boin et al., 2017)

\subsection{Sensoriamento Remoto}

Segundo Meneses (2012), o sensoriamento remoto é uma tecnologia com a capacidade de obter imagens de 
objetos da superfície terrestre sem que haja o contato físico entre os sensores e o objetos. Essa tecnologia captura ondas eletromagnéticas refletidas a partir da superfície da terra com a finalidade de observar e estudar a superfície terrestre e outros meios que refletem as ondas eletromagnéticas, como os oceanos. Os dados coletados com o uso do sensoriamento remoto podem ser de várias maneiras, incluindo variação na distribuição de forças, distribuição de ondas acústicas ou distribuição de energia eletromagnética (Lillesand et al., 2007).

\subsubsection{Aspectos físicos}

As radiações eletromagnéticas (REM) que são captadas dos sensores podem variar em relação ao comprimento de onda. No espectro visível ao olho humano, pode-se encontrar a variação de aproximadamente 400 a 700 nanômetros de comprimento (Moraes, 2002). O espectro eletromagnético compreende todas as variações de frequência, pode se dividir em: Raios gamas, Raios X, Ultravioleta, Visível (luz), Infravermelho, Micro-ondas e Rádio. A Fig. 1 mostra o espectro das ondas eletromagnéticas de acordo com o seu comprimento.

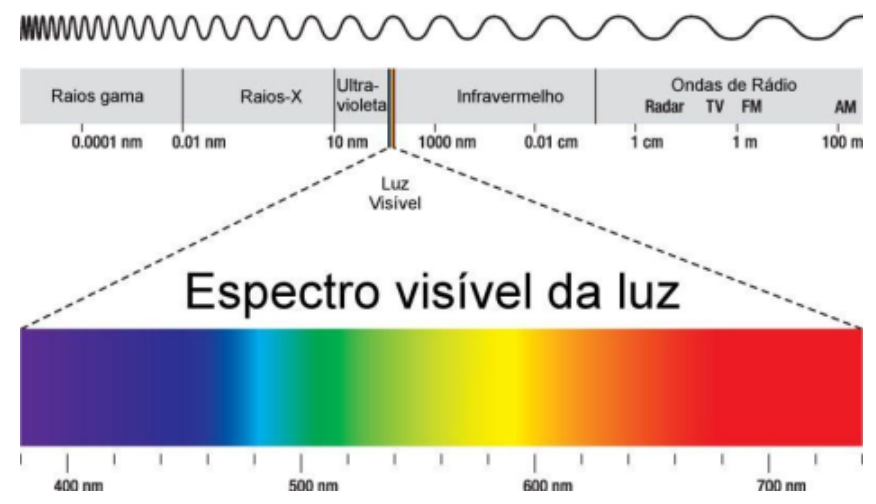

Figura 1: Espectro das ondas eletromagnéticas segundo seu comprimento

Quando um certo objeto possui alguma cor, quer dizer que este objeto reflete esta cor e absorve as REM equivalentes as cores restantes e isso depende das características moleculares do objeto. Objetos da superfície terrestre, como a vegetação, a água e o solo, refletem, absorvem e transmitem radiação eletromagnética em proporções que variam com o comprimento de onda, de acordo com as suas características biofísicas e químicas (Florenzano, 2007).

A visão humana consegue apenas reconhecer as REM que estão dentro do espectro visível. Os cones e bastonetes são células fotorreceptoras que estão no interior da região ocular do ser humano e absorvem ondas das REM, gerando informações ao cérebro. Os cones reconhecem apenas as cores vermelho, verde e azul. Já os bastonetes têm a capacidade de reconhecer a luminosidade. A combinação de cores dos cones é conhecida como teoria triestímulo da visão humana da cor, onde a combinação das cores captadas pelos cones formam todas as cores que enxergamos. No campo digital, foi cri- ada uma forma parecida de combinação muito utilizada em monitores e também no sensoriamento remoto, a combinação RGB ("Red, Green, Blue") (Meneses, 2012).

\subsubsection{Aplicações do Sensoriamento Remoto}

O uso de imagens de satélite torna possível se relacionar sob os aspectos multi-espectral, temporal e sócioeconômicos presentes na paisagem, como serras, planícies, bacias hidrográficas, matas e as regiões que tem a interferência humana, permitindo assim acompanhar a sua dinâmica, facilitando a compreensão entre as relações do homem com a natureza e suas consequências (Bonini, 2009).

O sensoriamento remoto pode ser aplicado em várias áreas, como a agricultura, geologia, recursos hídricos, estudos de ilhas de calor, etc. Diversas áreas de pesquisas utilizam o sensoriamento remoto para inúmeros tipos de estudos sobre uma determinada região, adquirindo assim um caráter multidisciplinar, garantindo vantagens para muitos profissionais e para a sociedade.

\subsection{O Sensor Infravermelho Termal}

O satélite Landsat- 8 contém um sistema imageador chamado Thermal Infrared Sensor (TIRS), as faixa térmicas fornecidas por este instrumento são úteis no fornecimento de temperaturas de superfície mais precisas. As duas bandas térmicas (TIRS) capturam dados com uma resolução mínima de 100 metros. Na Tabela 1 estão as características espectrais do dispositivo TIRS.

Tabela 1: Características espectrais do instrumento TIRS

\begin{tabular}{lll}
\hline Bandas & Comp. de onda $(\mu \mathbf{m})$ & Resolução $(\mathbf{m})$ \\
\hline Banda 10 - TIRS 1 & $10.60-11.19$ & 100 \\
Banda 11 - TIRS 2 & $11.50-12.51$ & 100
\end{tabular}

Os sensores do Landsat-8 fornecem uma melhor relação sinal-ruído (SNR) de desempenho radiométrico quantizado por meio de uma gama dinâmica de 12 bits. O sensor oferece melhor desempenho diante de ruídos, permitindo uma melhor caracterização do uso de cobertura do solo (USGS, 2019). Os tamanhos de arquivo Landsat-8 são maiores do que os dados do Landsat 7 , devido as bandas adicionais e o conjunto de dados aprimorados.

\subsection{Imagem digital}

As imagens digitais de sensoriamento remoto, contém valores numéricos proporcionais à intensidade de REM recebida e associada a cada pixel da imagem. Os tons de cinza variam de acordo com a intensidade de REM, variando do mais escuro (menor intensidade) ao mais claro (maior intensidade) (Carminato, 2015). A imagem da Fig. 2 mostra como são representados os pixels de uma imagem de uma banda vermelha.

As imagens Landsat contém 8 bits para cada pixel, portanto, o máximo valor numérico de um pixel destas imagens é 255, são todas as combinações possíveis de 


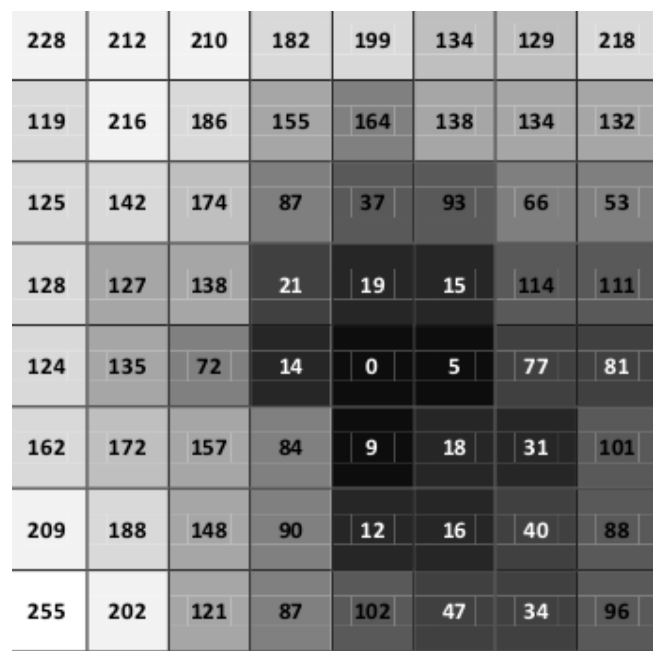

Figura 2: Um imagem matricial de uma banda vermelha

bits ligados e desligados. Cada sensor possui sua capacidade de definir o tamanho do pixel na imagem, onde o pixel diz respeito a menor parcela da imagem, sendo esta indivisível, esta dimensão do pixel é denominada resolução espacial (Rudorff et al., 2009). As imagens do Landsat têm resolução espacial de 30 metros. Quanto menor a dimensão do pixel, maior é a resolução espacial da imagem, possuindo uma maior definição dos objetos terrestres.

As imagens de sensoriamento remoto são fornecidas gratuitamente por meio de um registro do usuário no site do USGS. A partir dessas imagens pode-se realizar algum tipo de processamento em um Sistema de Informações Geográficas (SIG). As imagens estão separadas em diversas bandas, de acordo com o comprimento de onda de cada sensor, a combinação destas bandas deve ser feita conforme o estudo a ser realizado e as características da superfície terrestre que precisam ser destacadas, como, por exemplo, queimadas em uma região.

\subsection{Recorte de uma imagem matricial}

As imagens geoespaciais são, na verdade, matrizes multidimensionais muito extensas. O Sensoriamento Remoto, na sua forma mais simples, está realizando operações matemáticas nessas matrizes para extrair informações dos dados. Para o recorte de uma determinada região, deve-se possuir os arquivos de delimitação da região e o arquivo da imagem de satélite, assim que for recortada, o resultado deverá ser uma intersecção da máscara com o arquivo raster, como mostrado na Fig. 3.

As ferramentas que realizam as ações de recorte convertem o arquivo de delimitação de uma região no formato vetorial para uma matriz que pode ser usada como máscara, depois é carregado a imagem raster geoespacial em uma matriz; utilizando a máscara, em seguida são eliminadas todas as células da imagem que estão fora da extensão da máscara, definindo todos os
Shapefile Raster $\quad$ Resultado

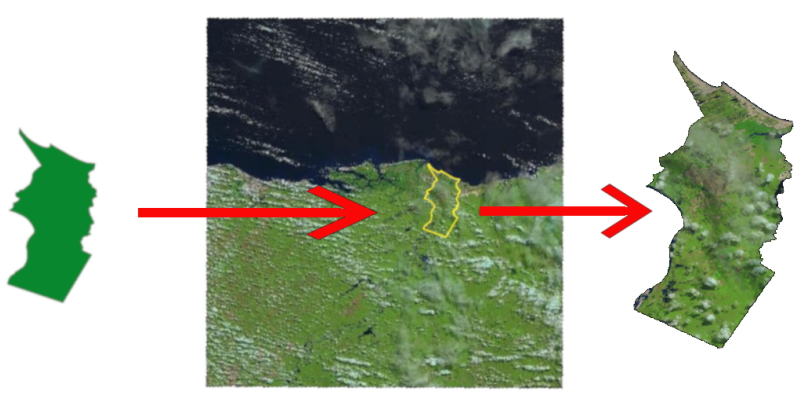

Figura 3: Recorte de uma imagem raster utilizando uma máscara

valores fora do limite como nulo, como passo opcional é realizado um alongamento do histograma na imagem para melhor visualização (Lawhead, 2015). Após isso é salvo a imagem resultante como uma nova imagem raster. O resultado final é uma imagem com apenas a região que foi gerada a partir da delimitação da região como molde de recorte, com base neste resultado, é possível realizar vários estudos sobre esta região delimitada.

\subsection{Acesso automatizado na web}

$\mathrm{O}$ acesso de dados automaticamente das páginas da Web tem a sua importância no que tange a obtenção de tipos específicos informações, sendo utilizados principalmente em motores de buscas e mineração de dados. A extração de dados de páginas Web geralmente é realizada por meio de agentes de software chamados Web crawlers que acessam de maneira automatizada a Web, navegando entre os recursos por meio de links, chamados de Web crawlers (Castillo, 2005). Um crawler executado sobre a Web tradicional trabalha com documentos HTML e links usando âncoras HTML, coletando esses documentos presentes na Web, como textos, imagens ou videos; armazenado esses dados em sistemas de armazenamentos próprios.

\subsection{Aplicações que utilizam imagens de Saté- lite}

Bruzelius et al. (2019) mostram que o uso de imagens de satélites coletadas em uma plataforma de acesso à essas imagens, somado à abordagens de análise de dados, podem ser aproveitadas para coleta de dados de populações remotamente assistidas e geograficamente isoladas, possibilitando uma maior tomada de decisão para agentes comunitários de saúde. Para Wulder et al. (2019), o uso de imagens do programa de satélites Landsat tem sido cada vez mais explorado por projetos governamentais, privados nacionais e internacionais com inúmeras aplicações como: monitoramento do desmatamento, mitigação das mudanças climáticas dentre outros; os autores ainda sustentam que os dados do satélite que são sistematicamente coletados e 
continuamente calibrados contribuem fortemente para a disseminação de técnicas que fazem o uso de geotecnologias. Sobre isso, Deijns et al. (2020), conseguiram detectar eventos naturais catastróficos utilizando processos semiautomatizados de obtenção de imagens do satélite Landsat, adicionando uma detecção mais rápida e escalável no tempo de detecção de distúrbios ambientais em grandes conjuntos de dados.

\section{Metodologia}

Este é um trabalho de pesquisa empírico de natureza qualitativa que descreve as etapas de implementação de um protocolo de aquisição e processamento automatizado do satélite Landsat-8. A definição do protocolo foi separado em três etapas: análise e levantamento de requisitos do protocolo manual, modelagem do banco de dados, implementação dos módulos e implementação da interface web.

Primeiramente foi definido cada etapa de aquisição e recorte de uma região específica na plataforma Earth Explorer, criando dois fluxogramas base, como apresentado nas Figs. 4 e 5.

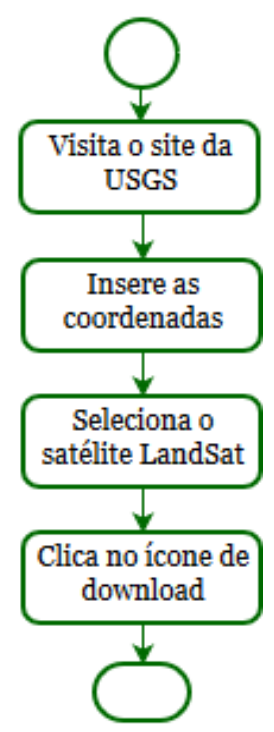

Figura 4: Fluxograma base de aquisição

O protocolo automático foi desenvolvido utilizando a linguagem de programação Python ${ }^{1}$. Foi escolhido o banco de dados PostgreSQL ${ }^{2}$ para a persistência dos dados que são manipulados pelo protocolo automático. Foram modelados quatro tabelas que representam cada entidade do método automático.

O modelo do banco de dados constitui de tabelas interligadas via chave estrangeira, a princípio as entidades foram implementadas seguindo um padrão de pedidos, onde há uma solicitação de um ou vários pedi-

\footnotetext{
$1_{\text {https: //www.python.org/ }}$

${ }^{2}$ https://www. postgresql.org/
}

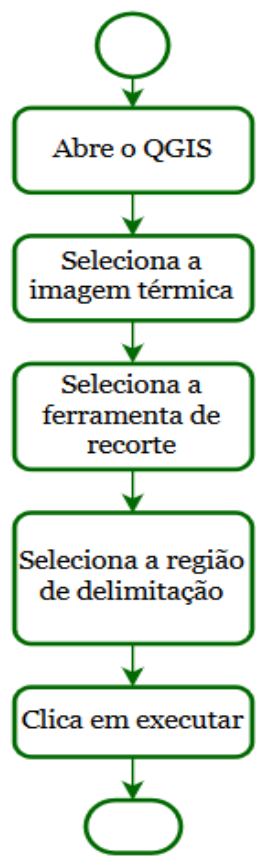

Figura 5: Fluxograma base de recorte

dos de regiões específicas, após isso o protocolo executa esses pedidos de forma assíncrona e periódica.

A Fig. 6 mostra a modelagem das tabelas que forma implementadas. A tabela a Scrapingorder que representa pedidos que serão solicitados para o protocolo automático, contendo uma relação com Raster e Coordinates. A tabela Raster identifica imagens de satélites adquiridas, já a Coordinates caracteriza informações de coordenadas de uma região, onde contém uma relação com a tabela Shapefile, que representa os arquivos de delimitação de uma região.

Os módulos foram implementados com o auxílio de bibliotecas de terceiros, merecendo destaque as seguintes ferramentas: Selenium ${ }^{3}$ que auxiliou a criação do web crawler para a coleta de dados na web da plataforma, o Celery 4 que adicionou a periodicidade e comportamentos assíncronos ao protocolo automático, o pygdal $^{5}$ que possibilitou a automatização do recorte de uma região e o PyDrive ${ }^{6}$ que viabilizou o upload e o download de arquivos no Google Drive.

Logo após a definição e implementação das entidades do banco de dados, foi verificada a estrutura da página web e o comportamento da plataforma da USGS em cada etapa presente no fluxograma base de aquisição, observando cada elemento da página que o usuário possa interagir para obter uma imagem térmica do Landsat-8. Com isso foi desenvolvido um script com o auxílio da ferramenta Selenium que automatizou a aquisição de uma faixa de foto do Landsat- 8 de acordo

\footnotetext{
$3 \overline{\text { https: //www.selenium.dev/ }}$

4http://www. celeryproject.org/

5 https://pypi.org/project/pygdal/

$6_{\text {https://pypi.org/project/PyDrive/ }}$
} 


\begin{tabular}{|c|c|}
\hline ScrapingOrder & Raster \\
\hline $\begin{array}{l}\text { + id: Integer } \\
\text { + coordinates: ForeignKey (id) } \\
\text { + raster: ForeignKey(id) } \\
\text { + status: Char(100) } \\
\text { + is_active: Boolean } \\
\text { + scraped_date: Date } \\
\text { + key: UUID }\end{array}$ & $\begin{array}{l}\text { + id: Integer } \\
\text { + acquisition_date: Date } \\
\text { + download_link: Char(100) } \\
\text { + file_id: Char(100) } \\
\text { + is_active: Boolean } \\
\text { + thumbnail_link: Char(100) } \\
\text { + key: UUID }\end{array}$ \\
\hline
\end{tabular}

*

\begin{tabular}{|l|l|l|}
\multicolumn{1}{|c|}{ Coordinates } & \multicolumn{1}{c|}{ Shapefile } \\
\cline { 1 - 1 } $\begin{array}{l}\text { + id: Integer } \\
\text { + shapefile: ForeignKey (id) } \\
\text { + is_active: Boolean }\end{array}$ & 1 & $\begin{array}{l}\text { + id: Integer } \\
\text { + is_active: Boolean } \\
\text { + key: UUID }\end{array}$ \\
\hline
\end{tabular}

+ title: Char(100)

+ latitude: Float

+ longitude: Float

+ description: Char(100)

+ is_active: Boolean

+ scraped_date: Date

+ key: UUID

Figura 6: Modelagem do banco de dados

com um ponto específico no mapa. A partir deste script foram criados outros quatro módulos para algumas funcionalidades do protocolo automático descritos a seguir:

- Spider: Busca a coordenada que foi informado por um pedido no banco de dados e visita a plataforma Earth Explorer automaticamente a fim de realizar o download do arquivo .zip que contém a imagem da região do satélite Landsat-8.

- Decompressor: Extrai a imagem térmica com o formato .tiff de um arquivo .zip baixada pelo módulo Spider, deletando os arquivos que não serão foco do trabalho.

- Trimmer: Este módulo faz o recorte de uma região específica de acordo com uma imagem de satélite com o formato .tiff e os arquivos de delimitação da região.

- Uploader: Faz o upload e download de um arquivo para a nuvem.

- Fetcher: Agenda novos pedidos para o protocolo automático.

Foi definido que as imagens baixadas pelo script que automatiza a aquisição seriam compactadas em um arquivo .zip; este arquivo deveria conter três imagens com a extensão .tiff, implicando apenas a extração da imagem térmica. Dessa forma foi aplicado os recursos do módulo de descompressão extraindo somente a imagem térmica de um arquivo zip. A periodicidade do protocolo automatizado, levou em conta a resolução temporal do Landsat-8. Com isso, para o auxílio na im- plementação do módulo Fetcher, as ferramentas Celery e Redis possibilitaram que a cada 16 dias fosse realizada uma execução agendada de uma nova coleta da região de interesse para o protocolo automático, considerando a data da última disponibilização da região a ser adquirida de um pedido presente no banco de dados. Os arquivos de delimitação foram organizados em três formatos, dentre os quais .shp, .dbf e .shx. Esses arquivos foram compactados com o formato .zip e submetidos na nuvem através de uma interface web para o upload deste arquivo. Por meio da biblioteca PyDrive que foi possível a disponibilização dos arquivos resultantes do processamento ao serviço de armazenamento na nuvem do Google Drive.

Foi estabelecido que a interação do usuário para a inserção das coordenadas e do arquivo .zip contendo os três tipos de arquivos seria por meio de uma interface web, desenvolvida com o auxílio do framework Django $2.0^{7}$. Foi adicionado a essa interface um formulário simples e um link com a lista de imagens que estão em processamento ou que já foram coletadas e recortadas.

O formulário contido na interface web faz a submissão dos pedidos para a coleta e recorte de uma região específica, sendo decidido que o mesmo deveria conter um título da região de interesse, uma descrição, as coordenadas latitude e longitude em formato decimal do ponto onde a região se encontra no mapa e, por fim, os arquivos comprimidos em formato .zip da região delimitada. Após a correta submissão do formulário, foi normatizado que a interface web submeteria um novo pedido para o protocolo automático. Ao finalizar os processos, o protocolo automático alteraria o status do pedido como encerrado usando a conexão com o banco de dados, agendando um novo pedido por meio do módulo Fetcher.

Foi definido que os pedidos do protocolo automático deveriam possuir basicamente quatro tipos de status: aguardando, executando, nenhum resultado, erro no pedido, concluído. Como as coordenadas de uma região foram determinadas como dois decimais; caso o web crawler não encontre nenhuma região com o ponto informado, por exemplo, um ponto onde fica no oceano, foi implementado que o protocolo automático atualizará o pedido para "nenhum resultado".

\section{Resultados e Discussão}

Como resultado dos ensaios de padronização do protocolo, foram obtidos resultados idênticos sobre uma região específica após aplicados os protocolos manual e automático. Além disso obteve-se diferentes imagens de uma mesma região em diferentes datas de acordo com a resolução temporal do Landsat-8. A região utilizada foi o município de Parnaíba, organizando seus dados de latitude, longitude e arquivo de delimitação em um arquivo de texto. A obtenção do arquivo de delimitação da região foi fornecida pela Secretaria do Meio Ambiente e Recursos Hídricos do Estado do Piauí (SEMAR).

\footnotetext{
7https://www.djangoproject.com/
} 


\subsection{Aquisição das imagens na plataforma EarthExplorer}

A plataforma EarthExplorer (EE) é uma ferramenta de busca, descoberta e solicitações online desenvolvida pela USGS. O EE suporta pesquisas de satélites, aeronaves e outros inventários de sensoriamento remoto por meio de recursos de consulta interativos e baseados em texto (USGS, 2019).

Foi visto que a plataforma possui vários meios para se coletar uma faixa de foto do satélite Landsat-8. 0 meio utilizado neste trabalho foi a inserção de coordenadas decimais de um ponto no mapa, mostrado na Fig. 7 .

\section{Degree/Minute/Second Decimal}

1. Lat: -2.9039 , Lon: -41.7763

Use Map Add Coordinate Clear Coordinates

Figura 7: Busca de uma região por meio de um ponto específico no mapa na plataforma EarthExplorer.

Após informado o ponto no mapa foi possível a aquisição de faixas de fotos que incluem a região onde o ponto se encontrava, selecionando algum dos variados datasets disponíveis. Neste trabalho foi escolhido o dataset "L8 OLI/TIRS", por fornecer as imagens com as bandas térmicas.

Com o dataset selecionado, a plataforma exibiu um conjunto de faixas de fotos onde o ponto estava contido, como mostrado na Fig. 8. O intervalo de busca de outra faixa de foto da região foi de 16 dias, sendo este intervalo estabelecido por ser exatamente a resolução temporal do satélite Landsat-8.

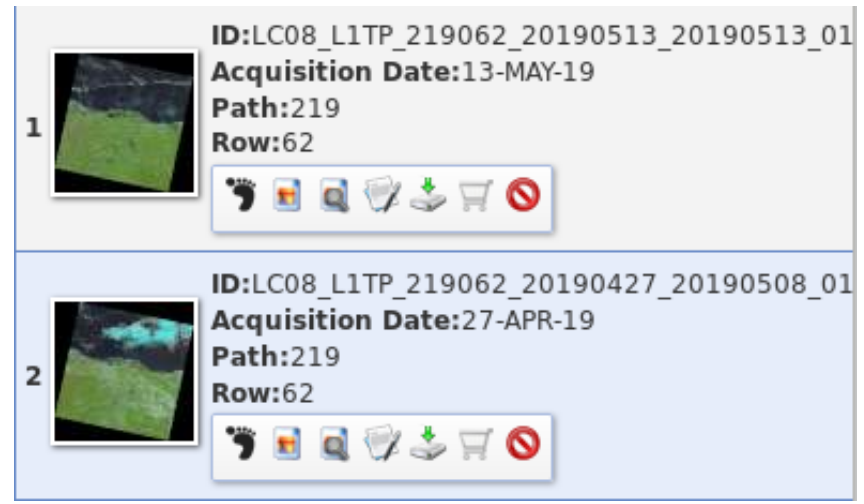

Figura 8: Lista de imagens de uma faixa de foto de uma região do Landsat-8

Cada foto contém opções de aquisição e visualização de informações, uma das informações mais importantes para o protocolo automático foi a data da disponibi- lização da imagem (acquisition date) na plataforma.

Foram encontradas na plataforma EarthExplorer (EE), cinco opções para a aquisição de uma imagem disponibilizada pela plataforma, neste trabalho foi escolhido a opção que contém as imagens com as referências geográficas, com as bandas térmicas 10 e 11 do sensor TIRS. O arquivo adquirido da plataforma continha não somente a imagem da faixa de foto, mas também diferentes bandas e os respectivos metadados.

\subsection{Recorte de uma imagem do Landsat-8}

Este trabalho utilizou o sistema geográfico QGIS para a verificação das ferramentas a fim de realizar o recorte de uma região específica, sendo um software de código aberto que contém uma interface para a linguagem Python e funciona em múltiplas plataformas (QGIS, 2019). Para o recorte de uma região foi necessário um arquivo do tipo shapefile que delimitou o contorno da região administrativa da cidade de Parnaíba, estado do Piauí, a qual foi definida como interesse do estudo.

Foi observado que para o protocolo manual a ferramenta apropriada disponível seria o QGIS para o recorte de uma região. Para o funcionamento deste recurso, foi adicionado como parâmetros os arquivos de delimitação e a faixa de foto contendo a região de Parnaíba, obtendo-se uma região específica como mostrado na Fig. 9.

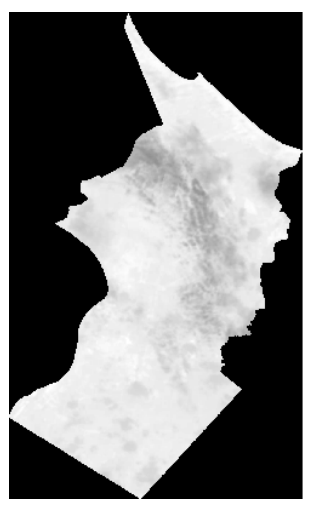

Figura 9: Recorte de uma região específica utilizando a ferramenta "clipper"do QGIS.

As imagens de satélites que foram obtidas estavam no formato Raster, ou formato matricial, contendo várias células com valores únicos, geralmente números ou rótulos textuais. A quantidade de células condiz com a resolução espacial da imagem, auxiliando na acurácia espacial.

O método para o recorte de uma imagem utilizando uma máscara, é adotado em grande parte dos trabalhos geográficos, pois esses estudos manipulam regiões específicas, tornando este tipo de pré-processamento extremamente importante. Já que o software QGIS continha uma interface com a linguagem de programação Python, dessa forma, para permitir uma maior adequação dos procedimentos de automatização com as 
ferramentas disponíveis, esta linguagem foi definida para o recorte automático da região.

\subsection{Resultados obtidos a partir da utilização do protocolo}

Utilizando as coordenadas da região de Parnaíba, foram obtidas regiões recortadas através do protocolo manual e automático. Observou-se que o resultado dos dois protocolos obtiveram exatamente a mesma saída, uma imagem térmica do satélite Landsat-8 do sensor TIRS de uma região específica. Na Fig. 10 é mostrado duas saídas de uma mesma região geradas a partir dos dois protocolos em períodos distintos, gerando a mesma região recortada mas com a coloração de tons de cinza diferenciados, comprovando que as fotos foram coletadas em períodos diferentes

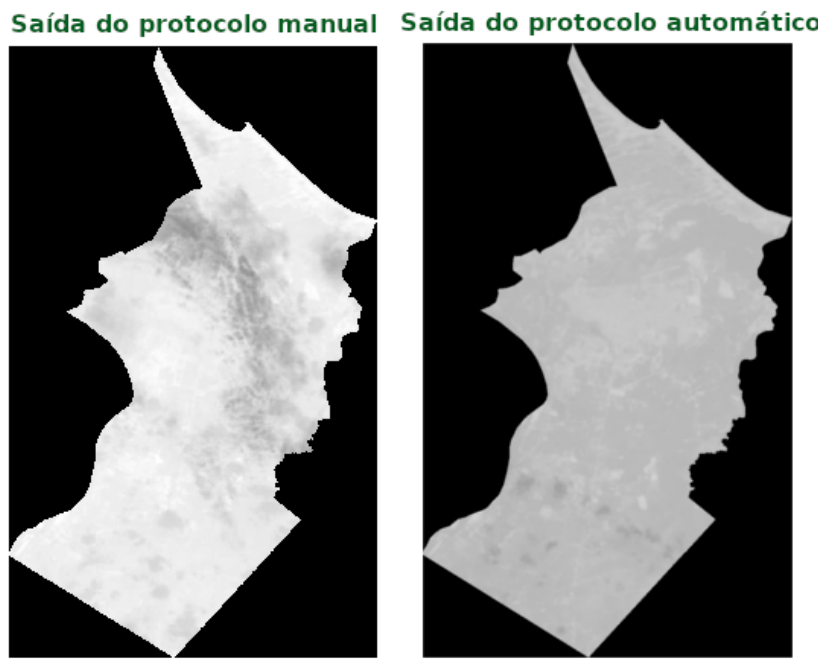

Figura 10: Saídas dos protocolos manual e automático

Foi visto como resultados preliminares que o web crawler não funcionou como esperado, ocasionado pela plataforma que exibia alertas no centro da tela em momentos variados, sendo necessário a adição de condições para a verificação destes alertas existentes no decorrer da visita do web crawler na plataforma. A princípio o web crawler seria implementado com o framework Scrapy, mas observou-se que a plataforma implementava códigos em javascript para sua página web, tornando inviável o uso do Scrapy, pois esta tecnologia não era compatível com o comportamento dinâmico encontrado em páginas web, sendo escolhido o uso do Selenium que contém recursos necessários para a interação automática com a página. Isso fez com que o protocolo automático ficasse um pouco mais lento do que o esperado, pois o Selenium custava alguns segundos para começar a interação com a página influenciando no desempenho do processo, porém, sem interferir em sua viabilidade.

\subsection{Resultados do protocolo automático}

Para avaliação dos procedimentos, Foi dado entrada ao protocolo automático os valores mostrados na Tabela 2, submetidos através de um formulário web. A data de submissão desses dados foi em 19/06/2019, adquirindo o primeiro resultado alguns minutos após a submissão do formulário. Com o término da coleta do primeiro resultado, o protocolo automático calculou o próximo dia da coleta de acordo com a data da última disponibilização da plataforma EE, sendo esta em 14/06/2019. Como a resolução temporal do Landsat-8 consiste em um intervalo de 16 dias, o protocolo automático calculou a coleta posterior para o dia 30/06/2019.

Tabela 2: Dados submetidos ao protocolo automático

\begin{tabular}{ll}
\hline Descrição & Valor \\
\hline Titulo & Recorte da região de Parnaíba \\
Latitude & -2.9039 \\
Longitude & -41.7763 \\
& zip(parnaiba_delimitacao.dbf, \\
Arquivo .zip & parnaiba_delimitacao.shp, \\
& parnaiba_delimitacao.shx)
\end{tabular}

Após 16 dias da data de disponibilização da imagem (30/06/2019), foi coletado o segundo resultado, substituindo a imagem antiga com a nova imagem coletada e calculando uma nova data de aquisição. Na Fig. 11 estão exibidos os dois resultados das aquisições obtidas via protocolo automático.

Região: Parnaíba, Latitude: -2.9039 , Longitude: -41.7763

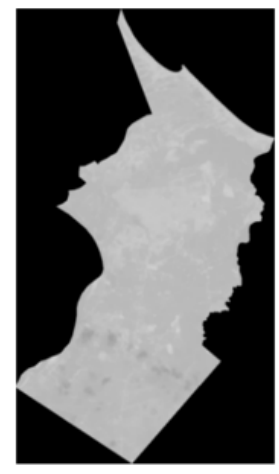

Disponibilização: 14/06/2019 Aquisição: 19/06/2019

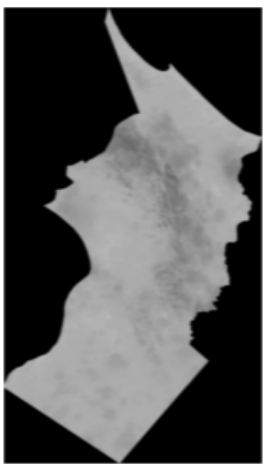

Disponibilização: 30/06/2019 Aquisição: 30/06/2019
Figura 11: Saídas do protocolo automático em diferentes datas 


\subsection{Arquitetura obtida}

Como procedimento, o protocolo automático recebeu como entrada de dados solicitações de pedidos, sendo estes encaminhados para o Celery para o processamento assíncrono e posterior agendamento automático a cada conclusão das tarefas.

Assim que o Celery obteve um pedido, instantaneamente uma tarefa assíncrona era instanciada e executada em um web crawler, utilizando os módulos que foram implementados para o pré-processamento da imagem. Assim que o arquivo foi adquirido automaticamente na plataforma do EE, novamente aplicou-se os recursos dos módulos Python para a extração da imagem do arquivo, submetendo a imagem extraída ao Google Drive em conjunto com banco de dados, para a persistência da imagem na nuvem e respectivas localizações no banco de dados local.

Notou-se que os pedidos que foram agendados e posteriormente desativados, não poderiam ser cancelados dinamicamente, pelo fato da ferramenta Celery não permitir esse tipo de operação, necessitando implementar uma condição no início da execução de um pedido para verificar se o mesmo não havia sido desabilitado, permitindo que a execução pudesse ser abortada caso o pedido não estivesse mais ativado no banco de dados.

Comparado com o protocolo manual, o protocolo automático fez com que o usuário não precisasse interagir com o Earth Explorer e o QGIS utilizando apenas o formulário e necessitando apenas a correta submissão com as entradas dos dados relacionados ao nome da cidade, descrição, coordenadas e arquivos de delimitação.

Com a automatização dos processos foram obtidos resposta mais rápida no que se refere a aquisição, recorte e disponibilização das imagens térmicas, otimizando o protocolo manual para ser realizado em poucas etapas.

\subsection{Representação do protocolo automático}

Com os passos definidos e feito a análise das tecnologias envolvidas no processo manual, foi possível a descrição do protocolo automático e implementação utilizando os módulos citados neste trabalho. A Fig. 12 apresenta um fluxograma com os principais passos do protocolo automático.

Como mostrado no fluxograma, as etapas com a marcação " $M$ "foram realizadas de maneira manual e as que contêm a marcação " $\mathrm{A}$ "definem os procedimentos automatizados definidos no protocolo. Sendo assim, foi necessário apenas a etapa manual de "inserção de coordenadas e do arquivo de delimitação"para a aquisição automatizada da imagem através do protocolo proposto. De forma complementar foi possível observar que o protocolo apresentado além de automatizar o processo, permitiu que a ação pudesse ser repetida iterativamente a cada passagem do satélite por uma determinada área e também a disponibilização da imagem em tempo real.

A implementação do protocolo automático teve auxílio da ferramenta git com disponibilização dos códigos na plataforma Github. O reposi-

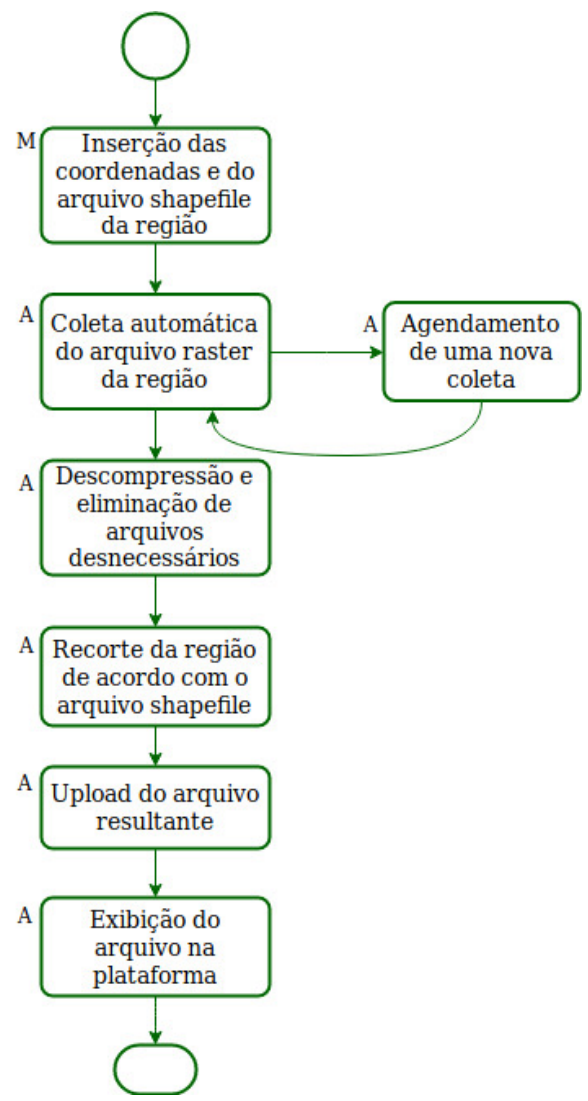

Figura 12: Fluxograma dos passos do protocolo automático

tório do protocolo automático foi disponibilizado com o título de LandSearch no seguinte endereço: https://github.com/AntLouiz/LandSearch.git.

\section{Conclusão}

Este trabalho teve a finalidade de definir um protocolo automático para aumentar o desempenho de pesquisas geográficas e verificar métodos de automatização de passos manuais em imagens de satélites. Dessa forma considera-se que:

- Foi possível automatizar a aquisição e o recorte de imagens térmicas do satélite Landsat-8.

- Não foi necessário, muitos recursos computacionais para a obtenção de uma região específica para o estudo de imagens térmicas.

- O protocolo permitiu acesso e plataformas que fornecem imagens e dados do satélite Landsat- 8 gratuitamente.

Com a disponibilização dados gratuitos na internet é inevitável o surgimento de novas tecnologias automáticas. A construção de ferramentas para o auxílio de estudos geográficos são de extrema importância, pois permitem um melhor planejamento e tomada de decisões. Essas ferramentas também podem ser combi- 
nadas, gerando outras tecnologias ainda mais robustas.

\section{Agradecimentos}

Gostaríamos de agradecer ao Centro de Geotecnologia (CGEO), coordenado pela Secretaria do Meio Ambiente e Recursos Hídricos do Estado do Piauí, na pessoa do Natanael de Araújo da Silva pelo apoio em disponibilizar o arquivo shapefile utilizado na execução dos ensaios desenvolvidos na pesquisa.

Agradecemos também à Universidade Estadual do Piauí, por disponibilizar o Laboratório de Processamento de Dados do Campus Profo Alexandre Alves e os recursos necessários para conclusão deste trabalho.

\section{Referências}

Boin, M. N., Martins, P. C. S. and Mirante, M. H. P. (2017). Geotecnologias aplicadas às questões ambientais.

Bonini, A. M. (2009). Ensino de geografia: utilização de recursos computacionais (Google Earth) no ensino médio. Disponível em http://hdl.handle.net/11449/104374.

Bruzelius, E., Le, M., Kenny, A., Downey, J., Danieletto, M., Baum, A., Doupe, P., Silva, B., Landrigan, P. J. and Singh, P. (2019). Satellite images and machine learning can identify remote communities to facilitate access to health services, Journal of the American Medical Informatics Association 26(8-9): 806-812. https://doi.org/10.1093/jamia/ocz111,.

Carminato, Larissa Porteiro Leite, G. T. D. e. S. V. D. P. (2015). Utilização de imagens de sensoriamento remoto em projetos de geoprocessamento, CONGRESSO ALICE BRASIL, Vol. 5.

Castillo, C. (2005). Effective web crawling, Acm sigir forum, Vol. 39, Acm, pp. 55-56.

Deijns, A. A., Bevington, A. R., van Zadelhoff, F., de Jong, S. M., Geertsema, M. and McDougall, S. (2020). Semi-automated detection of landslide timing using harmonic modelling of satellite imagery, buckinghorse river, canada, International Journal of Applied Earth Observation and Geoinformation 84: 101943. https://doi .org/10.1016/j.jag. 2019. 101943.

Fialho, E. S. (2009). Ilha de calor em cidade de pequeno porte: Caso de Viçosa, na Zona da Mata Mineira, PhD thesis, Universidade de São Paulo. https://www.doi.org/ 10.11606/T.8.2009.tde-22022010-154045.

Florenzano, T. G. (2007). Iniciação em sensoriamento remoto, Oficina de textos.

Lawhead, J. (2015). QGIS python programming cookbook, Packt Publishing Ltd.

Lillesand, Kiefer and Chipman (2007). Remote sensing and image interpretation. malden, ma.
Matias, L. F. (2001). Sistema de Informações Geográficas (SIG): teoria e método para representação do espaço geográfico, $\mathrm{PhD}$ thesis, Universidade de São Paulo.

Meneses, Paulo Roberto, T. d. A. e. o. (2012). Introdução ao processamento de imagens de sensoriamento remoto, Universidade de Brasília, Brasília .

Moraes, E. C. (2002). Capítulo 1 fundamentos de sensoriamento remoto. Disponível em http://mtc-m12.sid.inpe.br/col/sid.inpe.br/ sergio/2005/06.14.12.18/doc/CAP1_ECMoraes.pdf.

Mozgovoy, D. K., Svinarenko, D. N., Leong, Y. R., Zhigalov, K. Y., Tsarev, R. Y., Yamskikh, T. N. and Bystrova, N. V. (2019). Automated detection of deforestation based on multi-spectrum satellite data, Journal of Physics: Conference Series 1399: 044101. https: //www. doi.org/10.1088/1742-6596/1399/4/044101.

Nascimento and Oliveira (2011). Análise da evolução do fenômeno de ilhas de calor no município de goiâniago (1986-2010), Boletim goiano de geografia 31(2): 113127. https://doi.org/10.5216/bgg.v31i2.16849.

QGIS (2019). Um Sistema de Informação Geográfica livre e aberto. Disponível em https://www.qgis .org/en/ site/.

Rao, P. K. (1972). Remote sensing of urban"heat islands"from an environmental satellite, Bulletin of the American meteorological society 53(7): 647-648.

Rudorff, Theodor, B. F., MP, M., Shimabukuro and Edemir, Y. (2009). Imagens de satélites de sensoriamento remoto no brasil, Simpósio de Geotecnologias no Pantanal (GEOPANTANAL) 2: 1077-1083.

Sousa and Júnior, F. (2012). Relação entre temperatura de superfície terrestre, índices espectrais e classes de cobertura da terra no município de goiânia (go), Raega-O Espaço Geográfico em Análise 26. http://dx. doi.org/10.5380/raega.v26i0.30151.

Souza, S. O. (2015). Geotecnologias aplicadas à análise espaçotemporal do uso e da ocupação da terra na planície costeira de caravelas (ba) 1, Boletim Goiano de Geografia 35(1): 71-89. Disponível em: https:// www . redalyc . org/articulo . oa? id=337138459006.

USGS (2019). U.S. Geological Survey / Serviço de Levantamento Geológico Americano. Disponível em https: //www.usgs.gov/.

Wulder, M. A., Loveland, T. R., Roy, D. P., Crawford, C. J., Masek, J. G., Woodcock, C. E., Allen, R. G., Anderson, M. C., Belward, A. S., Cohen, W. B. et al. (2019). Current status of landsat program, science, and applications, Remote sensing of environment 225. https://doi.org/10.1016/j.rse.2019.02.015. 